\title{
Algebras of Hamieh and Abbas Used in the Dirac Equation
}

\author{
Gregory Peter Wene \\ Department of Mathematics, The University of Texas at San Antonio, San Antonio, USA \\ Email: gwene@utsa.edu
}

Received June 16, 2012; revised July 26, 2012; accepted August 3, 2012

\begin{abstract}
Hamieh and Abbas [1] propose using a 3-dimensional real algebra in a solution of the Dirac equation. We show that this algebra, denoted by $G \mathbb{C}$, belongs to a large class of quadratic Jordan algebras with subalgebras isomorphic to the complex numbers and that the spinor matrices associated with the solution of the Dirac equation generate a six-dimensional real noncommutative Jordan algebra.
\end{abstract}

Keywords: Dirac Equation; Jordan Algebra; Quadratic Algebra

Non-associative algebras have long been used in the mathematical description of physical phenomena; first appearing as the " $r$ number algebra" in the seminal paper by Jordan, Wigner and von Neumann [2] of 1934. The $r$ number algebra became known as a Jordan algebra from a 1946 paper by Albert [3]. The interested reader is referred to the books on non-associative algebras in physics Lõhmas, Paal and Sorgsepp [4], Okubo [5]. A concise history of non-associative algebra is to be found in Tomber [6]; the standard introduction to non-associative algebra is the book by Schafer [7].

Hamieh and Abbas [1] present a "description of an algebra which can be used in a possible extension of local complex quantum field theories". We further expand their description and show that these algebras are Type D Jordan algebras (see Jacobson [8]).

We construct a large family of quadratic Jordan algebras that contains the three-dimensional real algebra, the so called $G \mathbb{C}$ algebra, the generalized complex numbers, of Hamieh and Abbas [1], and show that the spinor matrices that arises from using the $G \mathbb{C}$ in a formulation of the Dirac equation generate a six-dimensional noncommutative quadratic Jordan algebra.

\section{Introduction}

Let $\mathfrak{A}$ be an algebra over a field $F$ not of characteristic two. The associator is a trilinear mapping

$$
(x, y, z)=(x y) z-x(y z)
$$

of $\mathfrak{A} \times \mathfrak{A} \times \mathfrak{A}$ into $\mathfrak{A}$ that measures the lack of asso-

${ }^{*}$ This paper is in final form and no version of it will be submitted for publication elsewhere. ciativity in $\mathfrak{A}$.

One scheme of classifying nonassociative algebras involves placing conditions on the associator of certain sets of elements. Some of the better known algebras are:

1) Alternative algebras. In this variety of algebras, all elements $x$ and $y$ satisfy

$$
(x, x, y)=(x, y, y)=0
$$

for all elements $x$ and $y$. The octonion division ring is an alternative algebra. An interesting variation is psuedooctonion algebra (Okubo [5,9]).

2) Jordan algebras. These are commutative algebras in which all $x$ and $y$ satisfy

$$
\left(x, y, x^{2}\right)=0 .
$$

A Type D Jordan algebra is the Jordan algebra of the symmetric bilinear form $q$ on a vector space $\mathfrak{B}$. Albert [3] has shown that any algebra of Type D has a basis $\left\{e, b_{1}, b_{2}, \cdots, b_{n}\right\}$ with multiplication given by

$$
\begin{gathered}
e b_{i}=b_{i} e=b_{i}, \text { for all } 1 \leq i \leq n, \\
b_{i} b_{j}=\delta_{i j} \alpha_{i} \text { for } 1 \leq i, j \leq n .
\end{gathered}
$$

The algebra will be semisimple if $\alpha_{i} \neq 0$ for all $1 \leq i \leq n$.

3) Noncommutative Jordan algebras. A generalization of the alternative and Jordan algebras that requires all $x$ and $y$ satisfy a generalization of the commutative law

$$
(x, y, x)=0,
$$

that is, the algebras are flexible, and

$$
\left(x, y, x^{2}\right)=0 \text {. }
$$


The book by Zhevlakov, Slin'ko, Shestakov and Shirshov [10] provides a detailed analysis of the alternative and Jordan rings.

The above algebras are all power associative since each element $a$ generates an associative subalgebra; equivalently, $a^{m} a^{n}=a^{n+m}$ for positive integers $m, n$. In any power associative algebra $\mathfrak{A}$ with unit element we can introduce the series

$$
e^{x}=\sum_{i=0}^{\infty} \frac{x^{i}}{i !}
$$

for $x \in \mathfrak{A}$ ignoring the question of convergence.

An algebra $\mathfrak{A}$ over a field $F$ is called quadratic if, for every $x$ in $\mathfrak{A}$

$$
x^{2}-2 t(x)+q(x) e=0
$$

where $t(x), q(x)$ are in $F$ and $e$ is the identity of $\mathfrak{A}$. The quantities $t(x)$ and $q(x)$ are called the trace and norm of the element $x$, respectively. The trace is a linear functional on $\mathfrak{A}$ see Schafer [7]. The norm $q(x)$ defines a symmetric bilinear form $q(x, y)$ on $\mathfrak{A}$ via

$$
q(x, y)=q(x+y)-q(x)-q(y) .
$$

Say $q(x)$ is nondegenerate if $q(x, y)$ is. Any quadratic algebra is power associative and any flexible, quadratic algebra is a noncommutative Jordan algebra.

A quadratic algebra $\mathfrak{A}$ is flexible if and only if the trace is associative; that is, $t((x y) z)=t(x(y x))$ for all $x, y, z$ in $\mathfrak{A}$. If $\mathfrak{A}$ is flexible then the mapping $x \rightarrow \bar{x}=2 t(x) e-x$ is an involution in $\mathfrak{A}$ (see Braun and Koecher [11], p. 216).

Lemma 1. The Hamiltonian division ring is a quadratic algebra.

Proof. Let $x=a+b I+c J+d K$ be an element of the Hamitonian division ring. Direct computation shows that

$$
x^{2}-2 a x+a^{2}+b^{2}+c^{2}+d^{2}=0 .
$$

Example 1. The octonion division ring is a quadratic algebras.

Example 2. Domokos and Kövesi-Domokos [12] propose a quadratic algebra, the "algebra of color" as a candidate for the algebra obeyed by a quantized field describing quarks and leptons (see also Wene [13,14], and Schafer [15]).

\section{Construction of the Algebras}

The elements of the algebra $G \mathbb{C}$ are the elements of the real vector space with basis $\{e, I, J\}$. The addition is the vector space addition and multiplication is defined by $I J=J I=0, I^{2}=J^{2}=-e, e$ is the identity and the distributive laws. We note that the algebra is commutative and has divisors of zero.

An immediate generalization of this algebra has a basis $\left\{e, b^{i}, i=1, \cdots, n\right\}, n \geq 2$ over the field $\mathbb{R}$ of real numbers and multiplication defined by $b^{i} b^{j}=-\delta_{i j}$ where $e$ is the identity. For want of a better name called these the Abbas algebras. As noted above, these algebras are Type D Jordan algebras. Note that the $G \mathbb{C}$ algebra is the construction for $n=2$; the results for the Abbas algebras apply to the $G \mathbb{C}$. Each Abbas algebra contains a copy of the complex numbers.

Lemma 2. The Abbas algebras are quadratic algebras.

Proof. Let $H$ denote a Abbas algebra. Then if $x \in H$, $x=\alpha_{0} e+\alpha_{i} b^{i}$, Einstein summation convention where $i=1,2, \cdots, n$. Then

$$
\begin{gathered}
x^{2}=\alpha_{0} x+\alpha_{0} \alpha_{i} b^{i}-\left(b^{i} b^{i}\right) \\
-2 \alpha_{0} x=-2 \alpha_{0}^{2}-2 \alpha_{0} \alpha_{i} b^{i}
\end{gathered}
$$

Adding both sides gives

$$
x^{2}-2 \alpha_{0} x=-\alpha_{0}^{2}-\left(\alpha_{i} \alpha_{i}\right)
$$

and we see that $t(x)=\alpha_{0}$ and $q(x)=\alpha_{0}^{2}+\left(\alpha_{i} \alpha_{i}\right)$.

A commutative quadratic algebra will be a Jordan algebra.

Since the algebra is commutative the trace is associative; the norm is symmetric.

Lemma 3. The norm of a Abbas algebra is nondegenerate.

Proof. Let $H$ denote a Abbas algebra. Then if $x \in H$, $x=\alpha_{0} e+\alpha_{i} b^{i}$ is arbitrary and $d=\delta_{0}+\delta_{i} b^{i}$ is fixed, then

$$
\begin{aligned}
q(d, x)= & q(d+x)-q(d)-q(x) \\
= & \left(\delta_{0}+\alpha_{0}\right)^{2}+\sum_{i=1}^{n}\left(\delta_{i}+\alpha_{i}\right)^{2} \\
& -\left(\delta_{0}^{2}+\delta_{i} \delta_{i}\right)-\left(\alpha_{0}^{2}+\alpha_{i} \alpha_{i}\right) \\
q(d, x)= & 2 \delta_{0} \alpha_{0}+2 \delta_{i} \alpha_{i}
\end{aligned}
$$

\section{Special $G \mathbb{C}$ Algebras}

Hamieh and Abbas [1] pass to a representation of the point $q=a e+b I+c J$ of the algebra $G \mathbb{C}$ in spherical coordinates, $a=r \cos (\theta), b=r \sin (\theta) \cos (\varphi)$ and $c=r \sin (\theta) \sin (\varphi)$. The subalgebras, called special $G \mathbb{C}$ algebras and denoted by $S G \mathbb{C}$ are the subalgebras spanned by all elements in which the "azimutal phase angle $\varphi$ is constant". Each of these subalgebras is (isomorphic to) the complex numbers.

Lemma 4. The algebra $G \mathbb{C}$ is isomorphic to an algebra of two by two matrices

$$
\left[\begin{array}{cc}
a & b I+c J \\
b I+J & a
\end{array}\right]
$$

under the usual matrix operation of addition and multiplication. 
Proof. The straight forward verification that the mapping $\theta(a e+b I+c J)=\left[\begin{array}{cc}a & b I+c J \\ b I+J & a\end{array}\right]$ is an isomorphism is left to the reader.

Lemma 5. Each of the algebras $S G \mathbb{C}$ is isomorphic to the complex numbers.

Proof. We note that if $b=r \sin (\theta) \cos (\varphi)$ then $c=b\left(\frac{\sin (\varphi)}{\cos (\varphi)}\right)$ if $\cos (\varphi) \neq 0$. If $\varphi=\frac{\pi}{2}$ or $\frac{3 \pi}{2}$, then $q=a+c J$ and the subalgebra $S G \mathbb{C}$ is (isomorphic to) the complex numbers. Otherwise, $q=a e+b I+b s J U$ or $q=a e+b(I+s J)$ for some $s \in \mathbb{R}$. Let $X=\frac{I+s J}{\sqrt{1+s^{2}}}$, then

$$
X^{2}=\left(\frac{I+s J}{\sqrt{1+s^{2}}}\right)^{2}=\frac{-\left(1+s^{2}\right)}{1+s^{2}} e=-e .
$$

The multiplication, using the basis $\{e, X\}$ will be given by

$$
(a e+b X)(c e+d X)=(a c-b d) e+(a d+b c) X .
$$

\section{The Spinor Matrices}

The classical reference on spinors and wave equations is the book by Corson [16].

The associator spinor matrices are

$$
\begin{aligned}
& C_{t}=\left(\begin{array}{ll}
0 & J \\
J & 0
\end{array}\right), \quad C_{x}=\left(\begin{array}{cc}
0 & -J \\
J & 0
\end{array}\right), \\
& C_{y}=\left(\begin{array}{cc}
0 & -I \\
I & 0
\end{array}\right), \quad C_{z}=\left(\begin{array}{cc}
1 & 0 \\
0 & -1
\end{array}\right) \\
& x y=\left(\begin{array}{cc}
-b s-c t & b v I+c v J \\
\mathrm{~d} s I+\mathrm{d} t J & \mathrm{~d} v-e y-f z
\end{array}\right) \\
& (x y) x=\left(\begin{array}{cc}
-e b v+f c v & (-b s-c t)(b I+c J)+d[(b v I+c v J] \\
(\mathrm{d} v-e y-f z)(e I+f J) & b \mathrm{~d} s+c \mathrm{~d} t+d(\mathrm{~d} v-e y-f z)
\end{array}\right) \\
& y x=\left(\begin{array}{cc}
-e y-f z & (x b+\mathrm{d} y) I+(c x+\mathrm{d} z) J \\
e v I+f v J & \mathrm{~d} v-b s-c t
\end{array}\right) \\
& x(y x)=\left(\begin{array}{cc}
-b e v-c f v & (\mathrm{~d} v-b s-c t)(b I+c J) \\
(-e y-f z)(e I+f J)+(\mathrm{d} e v I+\mathrm{d} f v J) & d(\mathrm{~d} v-b s-c t)-e \mathrm{~d} y-f \mathrm{~d} z
\end{array}\right)
\end{aligned}
$$
can write the elements $x$ and $y$ of the associator $(x, y, x)$ as $\quad x=\left(\begin{array}{cc}0 & b I+c J \\ e I+f J & d\end{array}\right)$ and $y=\left(\begin{array}{cc}0 & y I+z J \\ s I+t J & v\end{array}\right)$.
Lemma 7. The algebra $S P(6)$ is flexible.

Proof. Because of the trilinearlty of the associator, we

where $1, I, J \in G \mathbb{C}$. Denoting the 2 by 2 identity matrix by $I_{2}$, these matrices satisfy

$$
\begin{aligned}
& C_{t}^{2}=-I_{2} \text { and }\left\{C_{\mu}, C_{v}\right\}=C_{\mu} C_{v}+C_{v} C_{\mu}=2 \delta_{\mu \nu} I_{2} \\
& \text { for } \mu, v=x, y, z \text {. }
\end{aligned}
$$

The spinor matrices generate a 6-dimensional real lgebra with elements

$$
\left\{\left(\begin{array}{cc}
a & b I+c J \\
e I+f J & d
\end{array}\right) a, b, c, d, e, f \in \mathbb{R}, I, J \in G C\right\}
$$

that contains the matrix representation of the $G \mathbb{C}$ algebra. Denote this algebra by $S P(6)$.

Lemma 6. The algebra $S P(6)$ is a quadratic algebra.

Proof. If $x=\left(\begin{array}{cc}a & b I+c J \\ e I+f J & d\end{array}\right)$ is an element of $S P(6)$, then

$$
x^{2}=\left(\begin{array}{cc}
a^{2}-b e-c f & (a+d)(b I+c J) \\
(a+d)(f I+c J) & d^{2}-b e-c f
\end{array}\right)
$$

$-2\left(\frac{1}{2}\right)(a+d) x=-\left(\begin{array}{cc}a^{2}+a d & (a+d)(b I+c J) \\ (a+d)(e I+f J) & a d+d^{2}\end{array}\right)$

Adding the left and right sides gives

$$
x^{2}-2\left(\frac{1}{2}\right)(a+d) x=\left(\begin{array}{cc}
-a d-b e-c f & 0 \\
0 & -a d-c b-c f
\end{array}\right)
$$

equation over the complex numbers is often written as

$$
\left(i \gamma^{\mu} \partial_{\mu}-m\right) \Psi=0
$$

utilizing the Einstein summation convention for $\mu=$ $x, y, z, t$. A more general form is, setting
Theorem 1. The algebra $S P(6)$ is a quadratic noncommutative Jordan algebra.

\section{The Dirac Equation}

We proceed as in Hamieh and Abbas [1]. The Dirac 


$$
\begin{gathered}
\gamma^{\mu}=C_{\mu}, H \Psi=\left(C_{\mu} \partial_{\mu}\right) \Psi=m \Psi \\
\left(C_{\mu} \partial_{\mu}-m\right) \Psi=0,
\end{gathered}
$$

where $\mu=x, y, z, t$.

Upon substituting the matrices for $C_{\mu}$ and simplifying we get

$$
\left(\begin{array}{cc}
\partial_{z}-m & \partial_{z} \\
J \partial_{x}+I \partial_{y}+J \partial_{t} & -\partial_{z}-m
\end{array}\right)\left(\begin{array}{l}
\Psi_{1} \\
\Psi_{2}
\end{array}\right)=0 .
$$

In dimensions $x$ and $t$, the solution is given by

$$
\Psi(x, t)=N\left(\begin{array}{c}
\frac{E+p}{m} \\
1
\end{array}\right) e^{J(p x-c t)}
$$

$p$ and $E= \pm \sqrt{p^{2}+m^{2}}$ are respect the momentum and energy. $N$ is a normalization factor.

\section{Conclusion}

We have shown that the $G \mathbb{C}$ algebra belongs to a large class of Jordan algebras and have examined a few of the algebraic properties of these algebras and, like the Jordan algebra and the algebra of color, there is a very rich mathematical structure to further explore.

\section{REFERENCES}

[1] S. Hamieh and H. Abbas, "Two Dimensional Representation of the Dirac Equation in Non-Associative Algebra," Journal of Modern Physics, Vol. 3, No. 2, 2012, pp. 184186. doi:10.4236/jmp.2012.32025

[2] P. Jordan, J. von Neumann and E. Wigner, "On an Algebraic Generalization of the Quantum Mechanical Formalism," Annals of Mathematics, Vol. 35, No. 1, 1934, pp. 29-64. doi: $10.2307 / 1968117$

[3] A. A. Albert, "On Jordan Algebras of Linear Transforma- tions," Transactions of the American Mathematical Society, Vol. 59, No. 3, 1946, pp. 524-555. doi:10.1090/S0002-9947-1946-0016759-3

[4] J. Lõhmas, E. Paal and L. Sorgsepp, "Nonassociative Algebras in Physics," Hadronic Press, Palm Harbor, 1994.

[5] S. Okubo, "Introduction to Octonion and Other NonAssociative Algebras in Physics," Cambridge University Press, New York, 1995. doi:10.1017/CBO9780511524479

[6] M. L. Tomber, "A Short History of Nonassociative Algebras,” Hadronic Journal, Vol. 1, 1979, pp. 1252-1387.

[7] R. D. Schafer, "An Introduction to Nonassociative Algebras," Academic Press, New York, 1966.

[8] N. Jacobson, "Structure and Representations of Jordan Algebras," American Mathematical Society, Providence, 1968.

[9] S. Okubo, "Pseudo-Quaternion and Pseudo-Octonion Algebras," Hadronic Journal, Vol. 1, 1978, pp. 1250-1278.

[10] K. A. Zhevlakov, A. M. Slin'ko, I. P. Shestakov and A. I. Shirshov, "Rings That Are Nearly Associative," Academic Press, New York, 1982.

[11] H. Braun and M. Koecher, "Jordan-Algeben," SpringerVerlag, Berlin, 1966. doi:10.1007/978-3-642-94947-0

[12] G. Domokos and S. Kövesi-Domokos, "The Algebra of Color," Journal of Mathematical Physics, Vol. 19, No. 6, 1978, pp. 1477-1481. doi:10.1063/1.523815

[13] G. P. Wene, "An Example of a Flexible, Jordan-Admissible Algebra of Current Use in Physics," Hadronic Journal, Vol. 1, 1978, pp. 944-954.

[14] G. P. Wene, "A Little Color in Abstract Algebra," The American Mathematical Monthly, Vol. 89, 1982, pp. 417419. doi: $10.2307 / 2321659$

[15] R. D. Schafer, "A Generalization of the Algebra of Color," Journal of Algebra, Vol. 160, No. 1, 1993, pp. 93-129. doi:10.1006/jabr.1993.1180

[16] E. M. Corson, "Introduction to Tensors, Spinors, and Relativistic Wave-Equations," Chelsea Publishing Company, New York, 1982. 